\title{
Proteome allocation and the evolution of metabolic cross-feeding
}

\author{
Florian Labourel*,1, Frédéric Menu ${ }^{1}$, Vincent Daubin ${ }^{1}$, and Etienne Rajon ${ }^{1}$ \\ ${ }^{1}$ Univ Lyon, Université Lyon 1, CNRS, Laboratoire de Biométrie et Biologie Evolutive UMR5558, \\ F-69622 Villeurbanne, France
}

December 17, 2021

\begin{abstract}
Metabolic cross-feeding (MCF) is a widespread type of ecological interaction where organisms share nutrients. In a common instance of MCF, an organism incompletely metabolizes sugars and releases metabolites that are used by another as a carbon source to produce energy. Why would the former waste edible food, and why does this preferentially occur at specific locations in the sugar metabolic pathway (acetate and glycerol are preferentially exchanged) have challenged evolutionary theory for decades. After showing that cells should in principle prioritise upstream reactions, we investigate how a special feature of these metabolites - their high diffusivity across the cell membrane - may trigger the emergence of cross feeding in a population. We explicitly model metabolic reactions, their enzyme-driven catalysis, and the cellular constraints on the proteome that may incur a cost to expressing all enzymes along the metabolic pathway. We find that up to high permeability coefficients of an intermediate metabolite, the expected evolutionary outcome is not a diversification that resembles cross-feeding but a single genotype that instead overexpresses the enzymes downstream the metabolite to limit its diffusion. Only at very high permeabilities and under restricted sets of parameters should the population diversify and MCF evolve.
\end{abstract}

Key words - Metabolism, Specialisation, Mechanistic fitness, Proteome optimisation, Gene expression

\section{Introduction}

Genetic diversification (Wright, 1949; Ayala et al., 1974) may occur when different ecological niches are encountered (Gause, 1932; Hardin, 1960; Levin, 1972), for instance when different carbon sources are available in the environment (Hermsen et al., 2015; Wang et al., 2019). What may at first glance sound puzzling - why not using all the available nutrients? - finds an explanation in physiological constraints or even absolute incompatibilities that make specialists of each resource outperform generalists (Hutchinson, 1961; Stearns, 1989; Michod, 2005; Sheftel et al., 2013; Baquero et al., 2021). Even more bewildering is

${ }^{*}$ Corresponding author: florian.labourel@univ-lyon1.fr 
the observation that diversification occurs in the presence of a single energetic resource (Helling et al., 1987; Lenski et al., 1991; Good et al., 2017). One finds a clear example in chemostats or controlled experimental systems in which glucose is continuously injected, where glucose consumers may evolve that release metabolites for others to use as a carbon source (Helling et al., 1987; Treves et al., 1998). This unidirectional by-product process is a form of metabolite cross-feeding (D'Souza et al., 2018; Smith et al., 2019), and its evolutionary underpinnings are still blurry (D'Souza et al., 2018; San Roman and Wagner, 2018).

In particular, the reasons why specific metabolites are more likely involved in cross-feeding remain unclear. Indeed, a large number of metabolites can be produced by a glucose-reliant genotype and constitute a viable single carbon source for a second genotype (a glucose reliant strain of Escherichia coli can theoretically produce up to 58 such useful metabolites for example, San Roman and Wagner, 2018). Yet only two metabolites are commonly reported as being traded in such cross-feeding interactions, namely acetate and glycerol. San-Roman and Wagner (San Roman and Wagner, 2020) have hypothesized that this preferential evolution could be due to shorter mutational paths, such that modifying the metabolic network to produce these interacting strains would require fewer mutations and could thus occur more readily under a neutral model of evolution. But their conclusion is that acetate or glycerol trades are no more likely than others to arise by mutation and evolve neutrally.

In fact, adaptation is often incomprehensible without considering the ability of an organism to perform a task as dependent on internal constraints. For example, fully expressing all enzymes along a metabolic pathway may incur a fitness cost, such that sacrificing a part of a pathway becomes beneficial (Doebeli, 2002; Pfeiffer et al., 2004). The cost of packing a cell with proteins is actually two-fold (Labourel and Rajon, 2021). First, producing enzymes incurs a direct energetic cost, approximately proportional to the sum of enzyme concentrations in the cell (Wagner, 2005; Lynch and Marinov, 2015; Kafri et al., 2016). Second, cell packing may disturb the diffusion of enzymes, thereby hindering metabolic efficiency (Dill et al., 2011; Blanco et al., 2018). Making quantitative predictions thus requires to combine realistic features of cells and competitive principles.

In a previous study, we have shown that the evolution of enzyme kinetic parameters and concentrations is contingent on their competition with other processes for their substrate (Labourel and Rajon, 2021). One of these competing processes may be leakage through the cell membrane, such that highly diffusive metabolites should be processed by more efficient or concentrated enzymes. The combination of this requirement for high concentrations, and the cost of an abundant proteome, could make these metabolites the preferential points of rupture in a metabolic pathway by favoring a reduction in expression for downstream enzymes.

Very few metabolites can diffuse through membranes, either because of their size or due to their electronic properties (Milo and Phillips, 2016). Such diffusion may be direct, as is the case for glycerol, or indirect when a non-diffusive metabolite spontaneously transforms into a diffusive one, as is the case 
with acetate (Orsi et al., 2009; Pinhal et al., 2019). In this work - see Figure 1 for an overview of the metabolic model - we first determine how cells should allocate their proteome and find that upstream reactions should be favored when selective pressures are rather similar along the pathway. We then assess the hypothesis that cross-feeding evolves in response to the high diffusion rates of metabolites insofar as it increases the selective pressure acting on downstream reactions and eventually becomes insurmountable. As stated above, removing a part of a pathway may reduce the cost of over-expressing enzymes; interestingly, a genotype that would only express these enzymes to feed on the intermediate metabolite would not have to reach concentrations so high since the metabolite would already be present in the environment - i.e. high diffusion, for a specialist of the intermediate metabolite, is therefore beneficial.

We use Adaptive dynamics to model the competition for resource(s), as the environment is shaped by the genotype(s) in a population - that controls the equilibrium frequencies of both the nutrient and the intermediate metabolite. We find that cross-feeding interactions only evolves at very high membrane permeabilities of the focal metabolite, compatible with diffusion rates reported for acetate or glycerol. We also find such evolutionary diversification between two specialist phenotypes to occur at moderate to high, but not low, levels of degradation of metabolites along the pathway, and that it is also promoted by toxicity or reversibility effects, although between a generalist strategy and a specialist feeding on the intermediate metabolite in that case.

\section{Optimal metabolic allocation and cell constraints}

\section{Evolution of the overall expression of metabolic enzymes}

We first assume that all enzymes have an equal concentration and consider its evolution. Increasing concentration enhances the efficiency of catalysis and thus the production of energy, but with diminishing returns. It also incurs costs, firstly due to the actual energy cost of making proteins, and secondly because high protein concentrations in the cell decrease the efficiency of reactions due to cell packing. The former is captured in our model by a linear cost inflicted to extra production, and the latter through a penalty on $k_{f}$, whose effect has been estimated (Blanco et al., 2018; Andrews, 2020) and modelled in previous studies (Dill et al., 2011) - see Model and (Labourel and Rajon, 2021).

To approach the case of reactions involved in the carbon cycle (Liebermeister et al., 2014), we consider a pathway comprised of 40 enzymes and initiated by facilitated diffusion through a transporter (Kotyk, 1967), where an energy unit is produced at each step in the process - see Figure 1. Reactions follow Michaelis-Menten kinetics (Briggs and Haldane, 1925) - and we also consider the case where they are reversible, see SM - where we set their kinetic constants to $\log _{10}\left(k_{f}\right)=7$ and $\log _{10}\left(k_{c a t}\right)=2.5$. These are high values relative to the average enzyme in a cell, but only slightly (one order of magnitude) above the median observed for enzymes involved in the central metabolism (Bar-Even et al., 2011). The local context of a reaction - including reaction reversibility and metabolite toxicity - may also affect metabolic efficiency, as captured by a linear degradation rate of each metabolite, $\eta$, in this instance of the model 


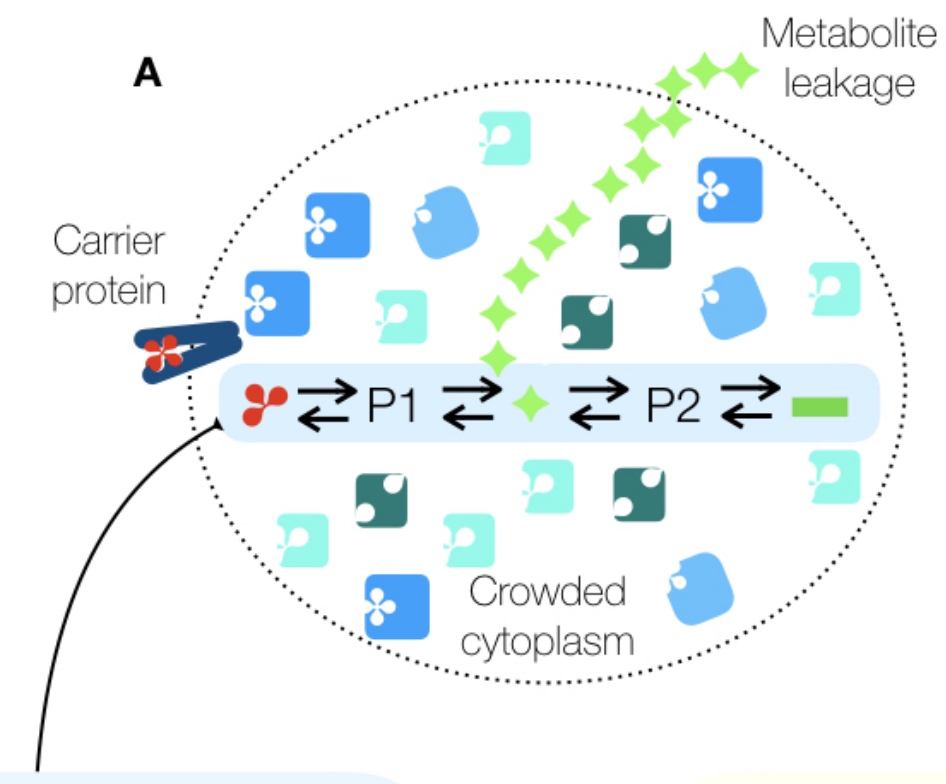

B

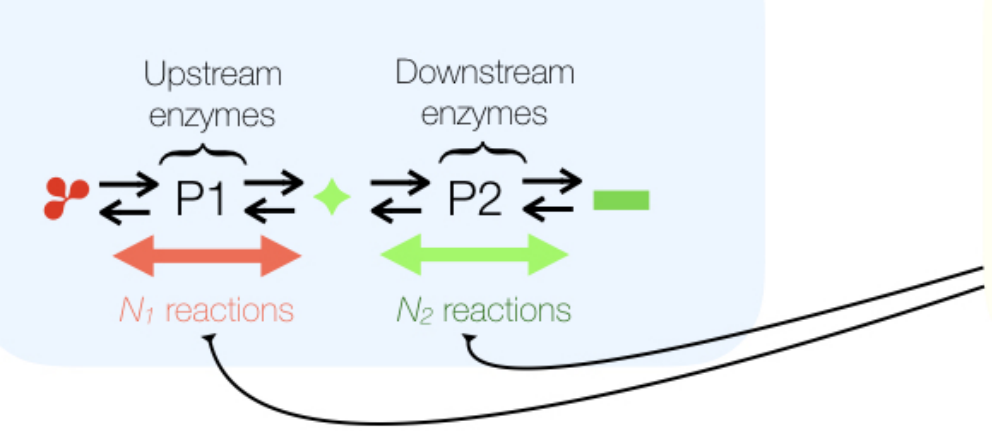

C Enzyme-substrate reactions

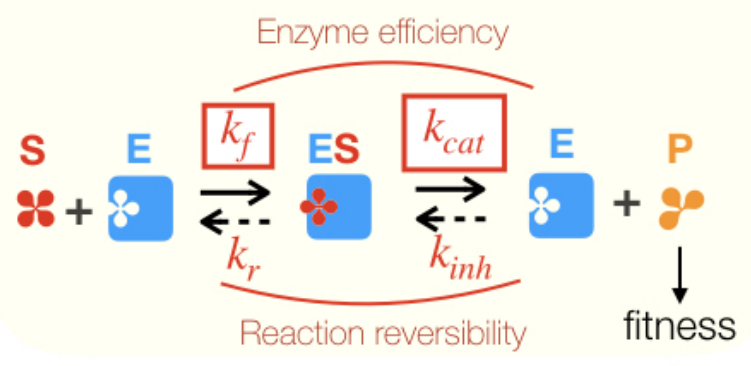

Figure 1: Overview of the model: the cell is packed with macromolecules, as shown in (A), to a point where any extra expression comes at a high cost so that the intracellular concentration reaches a ceiling no matter how this extra expression would influence the flux within a pathway (see first subsection of results for details). The pathway is initiated by a carrier protein and comprised of two sub-pathways P1 encompassing upstream enzymes, in red - and P2 - encompassing downstream enzymes, in green - whose respective lengths are $N_{1}$ and $N_{2}$ - see (B). Unless stated otherwise, these sub-pathways are of equal size and made of 20 enzymes each. These two sub-pathways are separated by a specific metabolite, whose leakiness varies from being very low to relatively high. Within this pathway, each reaction follows Briggs Haldane kinetics where enzyme efficiency is constant and studied as a parameter while reversibility varies depending on the subsection, and provides a fitness yield proportional to the amount of product produced and to the specific yield of energy gain set for reactions in the sub-pathway - see section on overexpression. Fitness is simultaneously impeded by the cost of expression and the toxicity induced by the total concentration of metabolites.

(Labourel and Rajon, 2021) - see SM for other types of cost. Nutrients are added to the environment at a constant rate $\alpha$ and degraded at a linear rate $\beta$ - set to physically realistic values in line with estimates for diffusive processes in the medium - which also applies to metabolites that can be released by cells.

For all combinations of the parameters above considered, the evolutionarily expected concentrations of the 40 enzymes in the pathway sum up to $15-25 \%$ of the whole proteome (see SM - section Text S1), according to Adaptive Dynamics - see Figure 2-A for an example of such convergent stable strategies (CSS, thereafter). Firmly logical, the highest fraction is obtained in conditions where selection for efficiency is 
acute, such that increasing concentration becomes beneficial, to a certain extent, despite amplifying intracellular crowding. These predictions are consistent with estimates among unicellular species (Liebermeister et al., 2014): in most cases, enzymes involved in the carbon cycle constitute 15 to $25 \%$ of the proteome. In the remaining of this study, the overall concentration of enzymes in the pathway is considered fixed at its evolutionary expectation by default, i.e. that obtained for the specific combination of parameters studied when assuming an identical allocation all along the pathway.
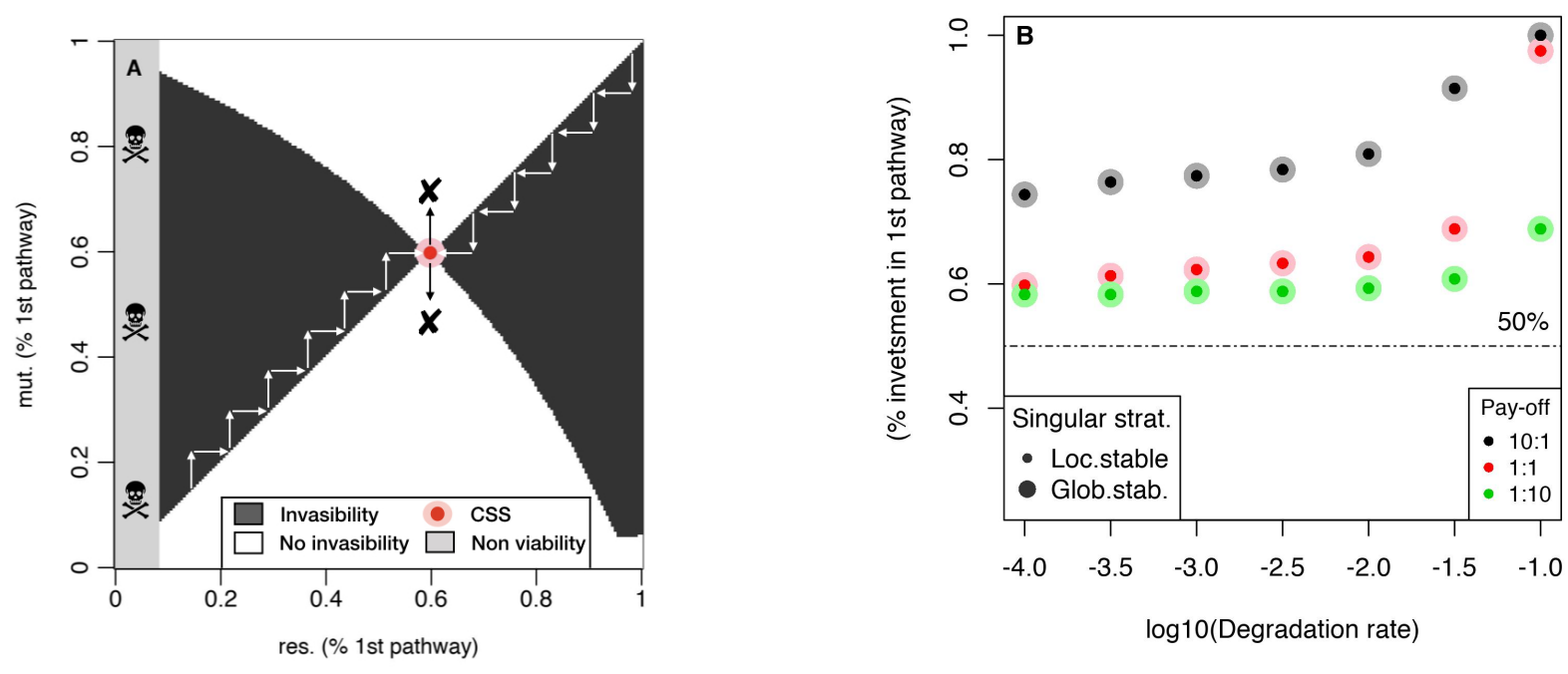

Figure 2: Figure A shows an example of pairwise invasibility plots, where black (resp. white) areas stand for positive (resp.negative) invasive fitness. The grey area represents an area where no strategy is viable because it cannot produce enough energy to reach the level set as corresponding to the demographic steady-state even when only one individual is present. When a mutant arrives in the population of resident strategies, it takes the place of the resident strategy if its invasion fitness is positive, a process represented by the white arrows. Here, below the CSS, mutants with higher values (black area above the left lower corner to right upper corner bisector) than the resident invades and evolution pushes resident to converge towards the CSS, while above the CSS, it is the other way around. On Figure B, we show how cells should spread their proteome content between upstream and downstream enzymes - referred to as $\delta$ in the text - when neither metabolite toxicity nor reversibility are considered (the influence of these factors is discussed in the text and shown in SM): in that case, it is always advantageous to invest more in upstream enzymes, an effect which decreases without completely vanishing when downstream reactions provide more energy than upwards ones - this is captured through the pay-off, with downstream reactions 10 times more fruitful than upstream ones when it is set to $1: 10$. Self-evidently, because the degradation rate eventually reaches levels for which the loss of metabolite becomes significant - see Figure S3 of SM to grasp this effect quantitatively - cells should prioritize even more the first part of the pathway.

\section{Overexpression in upstream reactions}

We then studied how cells should allocate their proteome along a pathway where several reactions are involved in energy production and fitness - for simplicity we consider that each reaction in either subpathway contributes equally. Because its selective pressure is mainly set by the transporter's features and therefore differs, the first enzyme in the pathway has a fixed concentration close to its optimal value, that is $\left[E_{0}\right]=10^{-4} M$, and does not contribute to fitness. We then split the rest of the pathway in two parts of equal length and study the evolution of the expression in each part. This is a proteome allocation problem, 
since the overall concentration is fixed as just described; we study the evolution of the part of this overall concentration allocated to the first half $P_{1}$ of the metabolic pathway, $\delta$, which we assume may change by mutation in the range $[0,1]$.

The evolution of $\delta$ is modelled using adaptive dynamics (Hofbauer and Sigmund, 1990; Geritz et al., 1998; Doebeli, 2002), as is appropriate when the fate of a mutant can depend on the environment shaped by one (or several) resident population. Here the resident strategy impacts the equilibrium concentrations of the nutrient and of the metabolites produced along the pathway. At this stage, each of these metabolites is considered identical to any other in the pathway, particularly regarding its diffusion across the membrane (i.e. membrane permeability for this metabolite is set to 0 ). Therefore in this case, the evolutionary outcome is always a single allocation strategy $\delta$, as shown in Fig. 2-A, where, from any resident strategy in place in the population, evolutionary trajectories will converge to $\delta \approx 0.6$, and, once in place in the population (as resident) this strategy will be stable against the invasion by mutants with any other $\delta$, which is known as a convergent stable strategy - CSS, hereafter.

The investment in the first part of the pathway is generally above $0.5:$ remarkably, $\delta$ evolves to approximately 0.6 even at very low degradation rates where the resulting loss in metabolites is far less than $1 \%$ along the pathway - see Figure S3 of SM, where we only report degradation rates above which the loss is significant, i.e. $\eta \geq 10^{-3} s^{-1}$. A plausible explanation is that upstream enzymes not only concur to fitness directly through the energy generated by their respective reaction, but also through their indirect contributions to downstream reactions - see Fig. 2 and SM Text S2 for the analysis of this phenomenon through a more tractable model. This is also consistent with the fact that this unequal allocation holds when downstream reactions produce far more energy than their upstream counterparts so that upstream reactions contribute mostly indirectly to fitness (and is significantly heightened in the opposite case): even when increasing the yield of the enzymes in the second half of the pathway tenfold as described for the carbon cycle (Cox and Nelson, 2000), $\delta$ stays close to 0.6. Besides, the irreversible loss of metabolites caused by an increase in the degradation rate (see Fig. S3 of SM) increases the asymmetry in fitness contributions further and thereby tends to increase the ratio of upstream to downstream enzyme expression (see Fig. 2). Such asymmetries in fitness contributions might help explain why enzymes catalysing more upstream reactions tend to face stronger selection (Greenberg et al., 2008; Wright and Rausher, 2010; Olson-Manning et al., 2012). Overexpression of upstream enzymes can nonetheless be - at least, partly - counteracted by selection for homogeneity in metabolite concentrations, as is the case when toxicity is high (Fig. 2B) and equally spread, and also depend on reactions reversibility, including that of the transporter: if downstream reactions are more reversible than upstream ones, cells should prioritise them, and vice versa. Be that as it may, considering realistic combinations of these pressures - moderate toxicity and degradation rates as well as the average reversibility found in central carbon metabolism (Li et al., 2011) - corroborate the need for upstream overexpression, as shown in Section Text S3.4 - see more specifically Figure S9. 


\section{Membrane permeability and cross-feeding}

\section{Membrane permeability impacts proteome allocation}

Membranes are only permeable to a few metabolites, owing to their unique chemical features (Milo and Phillips, 2016). In our model, we allowed the diffusion of the metabolite produced by the last reaction of the first half of the metabolic pathway - see the model overview on Figure 1 - with permeability rates ranging from $P=10^{-12} \mathrm{dm} . \mathrm{s}^{-1}$ to $10^{-4} \mathrm{dm} . \mathrm{s}^{-1}$ - in line with empirical estimates (Milo and Phillips, 2016), see the Model section for details. Metabolites making their way across the membrane have two effects. First, they are lost for the cell that has produced them, which may act as a selective pressure for limiting diffusion. Second, they may accumulate in the external environment and be available to other individuals as a resource. A cell has little leverage to limit outward diffusion; the most obvious solution is to use the metabolite before it is lost, which in our model is possible through an increase in the concentration of enzymes acting on the second part of the pathway. This is indeed what happens in our model: the optimal allocation shifts from a higher concentration in the first part of the pathway to a higher concentration in the second part as permeability increases as shown on Fig. 3 for the case of a low degradation rate (grey dots) : notice here that the enzyme following the transporter has the same concentration as any other one in the first sub-pathway as we also do not dissociate enzymes downstream the intermediate diffusive process, i.e. those of the second sub-pathway, inasmuch as reversibility should propagate, at least partly, the selective pressure modelled through the degradation rate - see previous section and SM section Text S3.4. The results presented in Fig. 3 are for proteins with kinetic parameters similar to the median of those reported for enzymes in the carbohydrate and energy pathway (Bar-Even et al., 2011). Higher efficiencies consistently produce a qualitatively similar result of a downward shift in allocation to the first part of the metabolic pathway $P_{1}$ as permeability increases (see Figure S5 in SM).

The reported large allocation to the second half of the pathway is a rather efficient strategy, associated with a slight decrease in the equilibrium concentration of the metabolite in the external environment (for diffusion rates above $10^{-8} \mathrm{dm} \cdot \mathrm{s}^{-1}$, see Fig. S5-B of SM). Despite its efficiency, this strategy results in an external concentration of the metabolite between $10^{-4}$ and $10^{-3} \mathrm{M}$, and is increasingly costly as permeability increases, as shown by the decrease in population size at high permeabilities - see Fig. S5-B of SM.

\section{High permeability coefficients can promote cross-feeding interactions}

This decrease in efficiency associated with an overinvestment in the second part of the pathway $P_{2}$, and the increase in the external concentration of the metabolite, may create a new ecological niche when genotypes increasing their contribution to the first or second part of the pathway become fitter than the generalist. These situations can be identified by a specific type of pairwise invasibility plots where the singular strategy is convergent but evolutionarily unstable, thereby defining a branching point as represented in Fig. 3-C. In these cases, an adaptive diversification may occur, which can then be studied through coalition invasion 

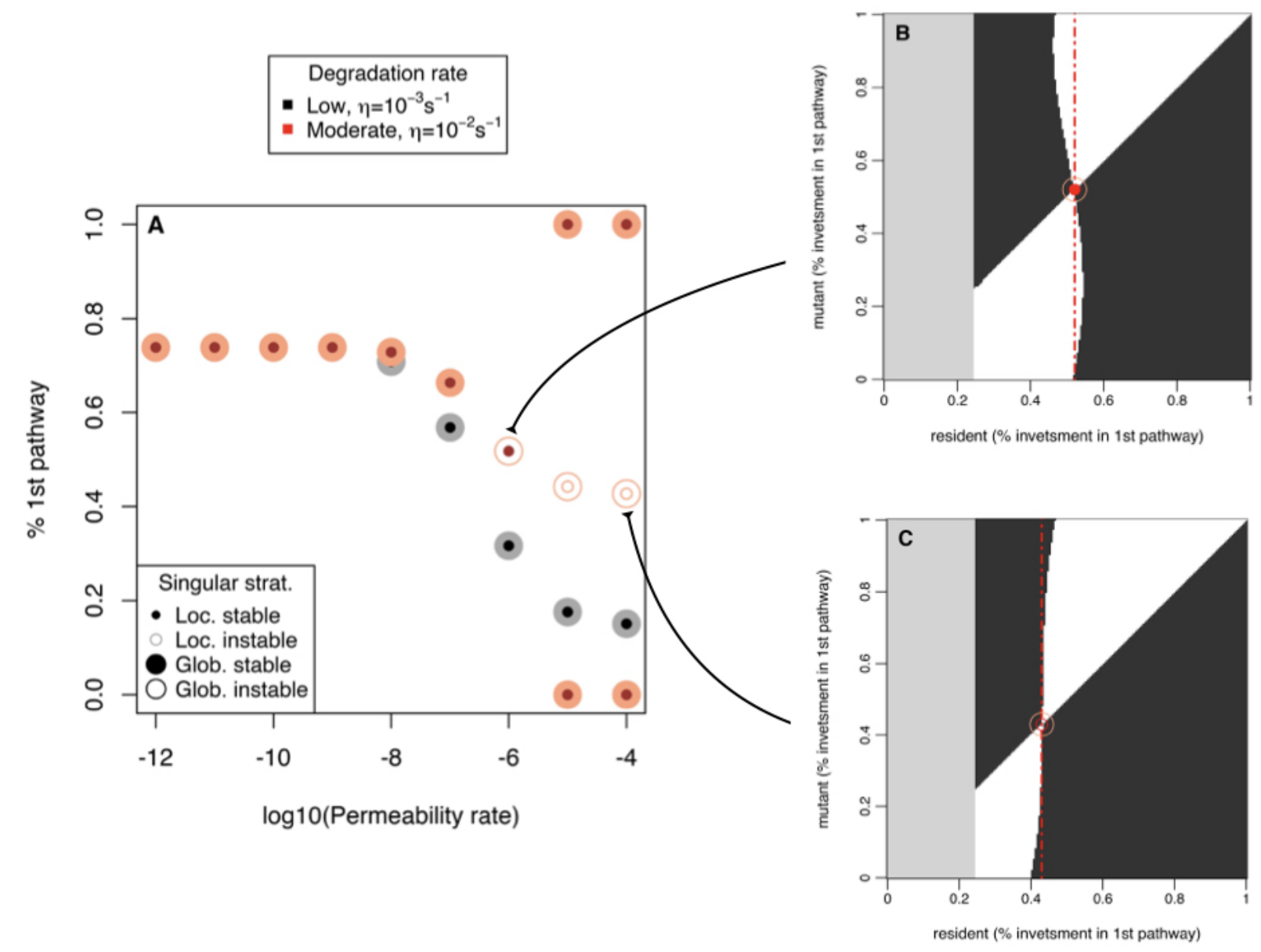

Figure 3: Permeability influences the evolution of strategies of enzyme allocations along a metabolic pathway, with the occurrence of cross-feeding at high permeability coefficients depending on the degradation rate. Two degradation rates are considered here (two others in Fig. S5) : low $\eta=10^{-3} \mathrm{~s}^{-1}$ and moderate $\eta=10^{-2} \mathrm{~s}^{-1}$. The enzymes catalysing reactions in either of two parts of the metabolic pathway, with their relative concentrations represented by $\delta$ (\% 1st pathway), have kinetic parameters typical of those intervening in the carbon cycle $\left(k_{f}=10^{6} \mathrm{M}^{-1} \cdot \mathrm{s}^{-1}\right.$ and $\left.k_{\text {cat }}=10^{2} \mathrm{~s}^{-1}\right)$; we also assume that reactions in the second part of the pathway produce 10 times more energy than reactions in the first part. For low permeability rates (below $10^{-6} \mathrm{dm} . \mathrm{s}^{-1}$ ), the evolutionarily expected strategy is always globally and locally stable - as in Figure 2-A - and consists in investing more in the first part of the pathway as described in Fig. 2-B. Increasing permeability coefficients above $10^{-} 7 \mathrm{dm} \cdot \mathrm{s}^{-1}$ results in a decrease in the investment in the first part of the pathway, $\delta$, with different consequences for low and moderate degradation rates. At low degradation rates, lowering $\delta$, i.e. increasing the investment in the second part, remains the most efficient strategy. At moderate degradation rates, however, high permeability coefficients result in singular strategies that are both convergent (they evolve from any starting $\delta$ ) and evolutionarily unstable (they can be invaded by mutants with close (locally unstable) or distant (globally unstable) $\delta$ (panel C). This can lead to adaptive diversification, resulting in a stable coalition of strategies, that is, a population made of coexisting genotypes with different values of $\delta$. The coalition can be determined (see SM -Section Text S3 for trait evolution plots) ; it is comprised of genotypes with $\delta=1$ (expressing only the first part of the pathway) and of genotypes with $\delta=0$ (expressing only the second part), corresponding to metabolic cross-feeding. B represents a permeability $P=10^{-6} \mathrm{dm} . \mathrm{s}^{-1}$ for which the singular strategy is globally instable but locally stable, so that the evolutionary outcome should be contingent to the mutational landscape.

graphs where the fate of mutants invading a pair of coexisting strategies is considered (SM Figure S6, section Text S3.1, and Appendix-Figure S14 and 15). When the degradation rate increases, the emergence of this new ecological niche eventually gives birth to such branching point, where the singular strategy is no longer stable (see $\mathrm{C}$ of Figure 3 for an extreme example where the singular strategy is both locally and 
globally unstable). For moderately high permeability rates - around $P=10^{-6} \mathrm{dm} \cdot \mathrm{s}^{-1}$ - the evolutionary outcome is actually dubious, but when this permeability maxes out at $P=10^{-4} \mathrm{dm} \cdot \mathrm{s}^{-1}$, cross-feeding diversification occurs between two specialists strategies, which was established by drawing trait evolution plots (see SM - Text S3.1 and Figure S15 for $P=10^{-5} d m \cdot s^{-1}$ ).

Diversification therefore occurs in our model when the degradation rate is moderate $\left(\eta>10^{-2} s^{-1}\right)$ and permeability is high (over $10^{-6} d m \cdot s^{-1}$; Fig. 3). The stable coalitions of strategies that evolve in this situation are composed of two phenotypically different genotypes: one that uses the nutrient as to produce the intermediate metabolite (corresponding to $\delta \approx 0$ ) and a second only consuming this metabolite $(\delta \approx 1)$. It should be highlighted, however, that the results in Figure 3 correspond to a metabolic pathway with unequal contributions of reactions to the energy needs of the cell (downstream reactions produce more than upstream ones, as observed on average in the carbon cycle). Equalling the energy contributions of all reactions often prevents the occurrence of cross-feeding. Yet, cross-feeding emergence was less sensitive to sub-pathway yields when we introduced toxicity and reversibility: in these contexts, diversification also occurs albeit between a generalist strategy and a cross-feeder specialised at the leaky metabolite (see Figures S8 and S10 of SM), coinciding with Black Queen dynamics often seen at the level of microorganisms (Jeffrey et al., 2012).

More generally, the evolution of cross-feeding seems quite contingent on several other factors: for instance, selection on the overall concentration of enzymes contributing to the pathway is important (for instance, by reducing diffusive constraints), such that allowing higher concentrations precludes the occurrence of cross feeding. This is because increasing downstream concentrations, which may efficiently deal with metabolite diffusion, comes at a lower cost on upstream concentrations under these circumstances. This illustrates that the occurrence of cross feeding may critically depend on the other tasks - and their contributions to fitness, which was not considered here - performed by cells and thereby on selection acting on their own proteome, as the global proteome concentration only varies to a small extent (Milo and Phillips, 2016). No less important should be the size of cells since smaller ones mechanically come with higher relative leakiness due to larger surface-to-volume ratios, which could favor the occurrence of cross-feeding. Cells can nonetheless adapt their size to their content, at least to a certain extent (Kafri et al., 2016), which may limit the costs incurred by an increase in concentration and prevent the occurrence of cross-feeding.

Finally, while in our model the efficiency of a reaction may only be changed through enzyme concentrations, kinetic parameters may also evolve and thus represent a relevant alternative in some specific parts of the metabolic network. The evolution of cross-feeding could thus also be contingent on the relative availability of mutations changing concentration versus kinetic parameters. Therefore, while this model includes much of the available information about enzyme kinetics and the selective constraints acting on the proteome, actually predicting how and when cross-feeding should evolve will require more efforts to better understand the building of global epistasis along metabolic networks. 


\section{Discussion}

The fact that few metabolites - acetate and glycerol, noticeably - are more likely involved in the evolution of cross-feeding has been a conundrum for as long as experiments have revealed this phenomenon (San Roman and Wagner, 2020). Here, we have put forward an explanation based on the necessity for a cell to optimise its proteome allocation, owing to its different incurred costs, and the existence of intrinsic constraints, noticeably the permeability of its membrane to a few metabolites. Indeed, acetate is in constant chemical equilibrium with the highly diffusive acid acetic (Pinhal et al., 2019), and glycerol readily leaks towards the environment (Milo and Phillips, 2016). The appearance of a new ecological niche when metabolites rather upstream in a metabolic pathway - and therefore of potential use to generate more energy - tend to diffuse, is not as straightforward as it may seem. In most cases that we have considered and that fall into the realistic range of parameters, proteome allocation will evolve in such a way that it prevents, or at least limits, the diffusion of the molecule. Only under some restricted conditions will cross-feeding evolve, characterized by a division of labor between a part of the population that transforms the nutrient into the diffusive metabolite, and another part that uses the metabolite as a carbon source, echoing work on digital evolution that similarly pointed to the possible contingency of cross-feeding (Meijer et al., 2020).

One process that may facilitate the emergence of cross-feeding, or that may be coopted to foster its efficacy when it is in place, is the export of metabolites through facilitated transport (Enjalbert et al., 2017; Pinhal et al., 2019). This process, where cells actively give up a metabolite even though it still has the potential to bring fitness contributions, is often known as overflow metabolism (Vemuri et al., 2006) and also often involves acetate. We did not consider the existence of a documented cost to the second part of the pathway, as has been documented in the past to explain overflow (Peebo et al., 2015; Basan et al., 2015), nor did we account for other possible costs such as the existence of a localised toxicity (Kemble et al.) or the membrane occupancy involved in cellular respiration (Zhuang et al., 2011; Szenk et al., 2017). These processes may promote the advent of cross-feeding, for they could bring extra fitness to an organism willing to specialise at one or the other part of the pathway. For instance, in a rich environment where producing energy is easy, it may be relevant to free some part of the membrane in order to sustain the uptake of other useful and otherwise costly nutrients. Yet, they are generally associated with rich environments while cross-feeding does not seem to be limited to these conditions. Besides, we believe that studying this question needs not bypass epistatic relationships stemming from the joint evolution between enzyme kinetic parameters (Heckmann et al., 2018) and their expression along pathways (Kryazhimskiy, 2021); this may yield unexpected predictions, since increasing expression - as we did here - is not identical to increasing kinetic efficiency.

In parallel, it is in fact also possible that cross-feeding only arises as the by-product of permeability without bringing any fitness advantage to its initiator. Because there is an unexploited niche, an organism could evolve that thrives on this metabolite, even at the cost of a slower pace of life. This may be even truer since nutrients are almost never constant in Nature, but rather more or less subject to stochasticity. What strategy should be favored in the light of such unpredictable fluctuations should be addressed to 
make more quantitative predictions: even a fast growing cell may indeed be willing to feed on the residual wasted product when its main source of food is about to or has already disappeared (Solopova et al., 2014; Enjalbert et al., 2017; Millard et al., 2021).Which strategy should evolve and whether it is conditioned by the environmental context will tell us more about the rules behind microorganisms community assembly (Goldford et al., 2018; Bajic and Sanchez, 2020; Estrela et al., 2020), a longstanding and ongoing question in evolutionary ecology. We believe that the present study will help explain how the organisms composing these communities appeared in the first place and how it may have fuelled microbial cooperative behaviours.

\section{Material \& Methods}

\section{Metabolic Model of fitness}

Cell fitness results from the biomass and energy produced along a metabolic pathway (eg. ATP). The pathway is initiated by carrier proteins, passively transporting nutrients inside cells and whose features are based on those for glucose in yeasts, as detailed in (Labourel and Rajon, 2021). Nutrient molecules are added (resp. degraded) at a constant rate $\alpha$ (resp. $\beta$ ) in the external environment. The metabolic pathway is linear, comprising a first reaction catalysed by an independent enzyme followed by 40 reactions catalysed by enzymes whose expressions is considered in this study. Each reaction follows either reversible or irreversible Michaelis Menten kinetics (Haldane, 1930):

$$
\mathrm{E}_{\mathrm{i}}+\mathrm{S}_{\mathrm{i}} \underset{\mathrm{k}_{\mathrm{r}}}{\stackrel{\mathrm{k}_{\mathrm{f}, \text { act }}}{\rightleftharpoons}} \mathrm{ES}_{\mathrm{i}} \underset{\mathrm{k}_{\text {inh, act }}}{\stackrel{\mathrm{k}_{\mathrm{cat}}}{\rightleftharpoons}} \mathrm{E}_{\mathrm{i}}+\mathrm{P}_{\mathrm{i}}
$$

where reversibility is spread equally between backwards paramaters $k_{r}$ and $k_{i n h}$ (eg. if $K_{\text {rev }}=1 / 9$, $k_{r}=k_{c a t} / 3$ and $k_{i n h, a c t}=k_{f, a c t} / 3$ with $k_{i n h}$ also being affected by crowding, which is a conservative assumption as reverse reactions should be less sensitive to the diffusive process owing to the preexisting co-localisation between substrate molecules and enzymes).

Each reaction produces energy - which is a simplification of the carbon cycle where some do not, unimportant as we consider the global expression of large portions of a pathway. We consider the case where contributions are equal along the pathway as well as other more realistic setups.

\section{Cellular constraints}

Cell proteomes face two intrinsic constraints: (i) the burden of protein expression and (ii) the cost entailed by molecular crowding. We model (i) through a linear cost $c$ impeding fitness (Lynch and Marinov, 2015). We considered values of $c$ such that the whole cytoplasmic proteome - the enzymes in the pathway and other free enzymes - costs 5 to $50 \%$ of the whole cell budget. We model molecular crowding (ii) through a non-linear decrease of diffusion (Dill et al., 2011; Blanco et al., 2018) that changes the affinity constant 
$k_{\mathrm{f}}$ to $k_{\mathrm{f}, \text { act }}$ according to equation:

$$
k_{\mathrm{f}, \mathrm{act}}=k_{\mathrm{f}} \cdot 10^{-\left(\left[E_{\mathrm{other}}\right]+\sum_{i=1}^{40}\left[E_{t o t, i}\right]\right) /\left[M_{b}\right]},
$$

where $\left[E_{t o t, i}\right]=\left[E_{i}\right]+\left[E S_{i}\right],\left[M_{b}\right]=3 \cdot 10^{-3} M$ represents the scaling factor for the effect of diffusion, while $\left[E_{\text {other }}\right]$ denotes the sum of the concentrations of other cytoplasmic proteins than the 40 under consideration.

Our model includes three processes involving the metabolites produced that select for the enhancement of enzyme activity, drawing a complex trade-off on the coexpression of enzymes: (1) metabolites can be lost, either because they are involved in parasitic reactions or because they are subject to targeted degradation (Peracchi, 2018), modelled through a linear degradation rate $\eta$; (2) metabolites can be toxic for the cell, for they engage in parasitic reactions, for instance through promiscuous interactions (Niehaus and Hillmann, 2020); (3) highly reversible reactions within a pathway may also require efficient enzymes to maintain a high net flux (Heinrich et al., 1991). We considered these three processes in various instances of our model, as described in SM Text S1; the results presented in the paper mainly comprise the action of a linear degradation rate, which provides a good qualitative understanding of how processes impacting the metabolites also impact selection on enzymes. Finally, the permeability of cell membrane to a given metabolite also acts as a constraint, which is introduced here by considering that one metabolite in the pathway diffuses passively at a rate $\eta_{d}$ - on Figure 1, we show where this process occurs.

\section{Adaptive Dynamics of enzyme expression}

We use Adaptive Dynamics (Hofbauer and Sigmund, 1990; Geritz et al., 1998; Doebeli, 2002) to model the evolution of enzyme expression along the metabolic pathway. This framework consider rare mutations, such that a resident "strategy" - corresponding to a given expression pattern - is assumed to have reached its demographic equilibrium before a mutant strategy appears in the population. At this demographic equilibrium, births compensate for deaths in the population, resulting in a concentration of nutrients specific of the resident (see next section). The fitness of any mutant strategy is then determined for each resident equilibrium, which enables the drawing of Pairwise Invasibility Plots (PIPs) representing for each pair the ability of a rare mutant to invade the resident strategy, based on a comparison between the growth rate of the mutant and 1 . These plots are used to identify singular strategies and their properties, as defined in (Geritz et al., 1998). A particular type of singular strategies, branching points, may be indicative of a diversification in the population, which we further study by drawing areas of mutual invasibility (each strategy invades when rare), and then computing the ecological equilibrium for each coalition - composed of two resident strategies instead of one - in that space. We then calculate the growth rate of mutants for each nearby mutants of each strategy in the coalition to identify coalitions that are stable (they cannot be invaded by any of the nearby mutants) and convergent (there exists evolutionary trajectories towards them), hence identifying evolutionarily expected communities (or bimodal populations) after diversification 
has occurred (Dieckmann and Law, 1996; Dieckmann and Doebeli, 1999). This latter process is summarised on trait evolution plots - TEPs - that we have shown in SM.

\section{Ecological equilibria}

As to draw pairwise invasibility plots, we determine the equilibrium through a three steps process (it is not possible to use solver methods on the whole system for they fail to find consistent solutions as a consequence of the huge difference in order of magnitude between metabolite concentrations and population size). First, we set an equilibrium flux - to $\Phi_{n e t, N_{e q}}=10^{-4} M$ which is needed to sustain the population at equilibrium. We then compute the value of the net flux $\Phi_{n e t, N}$ for a given population size $N$. As long as it is lower (or higher) than $\Phi_{n e t}$, population is increased (or decreased) at the next iteration. The algorithm is stopped either when the difference between these fluxes is lower than $10^{-6} \Phi_{n e t, N}$, or when it oscillates between two neighbouring values ( $N_{e q}$ is then set to the average between these values). Ecological equilibrium matches with the concentration in the environment for this demographic equilibrium. Notice that to make the algorithm quicker, the first 100 iterations change population size by the ratio between the current net flux and its targeted equilibrium value. Then, a fine adjustment is lead by increasing or decreasing the population size by one unit. The same process is used for coexistence, although henceforth applied to resident coalitions composed by two resident strategies that each has its own population size at equilibrium. PIPs were generally drawn for 250 strategies, unless lower resolution was sufficient to capture the trend. In order to determine the optimal allocation, we set the total proteome content to its optimal value as determined without the influence of permeability. An individual whose strategy is to invest as much in the first part of the pathway than in the second part corresponds precisely to this case. Notice that, besides using a two step ptocess, solving the systems to find nutrient and metabolite concentrations required to use R package 'nleqslv' with Broyden's method (Hasselman, 2018).

\section{Settings for the models}

A list of the basic settings can be found at the end of section Text S1.1 - SM. We varied them within their biological realistic ranges. This allowed us to identify key drivers of the diversification process that eventually result in cross-feeding, as discussed in the results section. The extensive analysis of parameters can be found in SM Texts S1 and S3.

\section{Data Archival}

We plan to make available the scripts used throughout this study through a git repository.

\section{References}

S. S. Andrews. Effects of surfaces and macromolecular crowding on bimolecular reaction rates. Phys Biol, 17(4):045001, May 2020. ISSN 1478-3975 (Electronic); 1478-3967 (Linking). doi: 10.1088/1478-3975/ab7f51. 
F. J. Ayala, M. L. Tracey, D. Hedgecock, and R. C. Richmond. Genetic differentiaiton during the speciation process in drosophila. Evolution, 28(4):576-592, Dec 1974. ISSN 1558-5646 (Electronic); 0014-3820 (Linking). doi: 10.1111/j.1558-5646.1974.tb00791.x.

D. Bajic and A. Sanchez. The ecology and evolution of microbial metabolic strategies. Current Opinion in Biotechnology, 62:123-128, 2020. doi: https://doi.org/10.1016/j.copbio.2019.09.003. URL https://www.sciencedirect.com/science/ article/pii/S0958166919300722.

F. Baquero, T. M. Coque, J. C. Galán, and J. L. Martinez. The origin of niches and species in the bacterial world. Frontiers in microbiology, 12:657986-657986, 03 2021. doi: 10.3389/fmicb.2021.657986. URL https://pubmed.ncbi.nlm.nih.gov/ 33815348.

A. Bar-Even, E. Noor, Y. Savir, W. Liebermeister, D. Davidi, D. S. Tawfik, and R. Milo. The moderately efficient enzyme: Evolutionary and physicochemical trends shaping enzyme parameters. Biochemistry, 50(21):4402-4410, 05 2011. doi: 10.1021/bi2002289. URL https://doi.org/10.1021/bi2002289.

M. Basan, S. Hui, H. Okano, Z. Zhang, Y. Shen, J. R. Williamson, and T. Hwa. Overflow metabolism in escherichia coli results from efficient proteome allocation. Nature, 528(7580):99-104, 12 2015. doi: 10.1038/nature15765. URL https: //pubmed.ncbi.nlm.nih.gov/26632588.

P. M. Blanco, J. L. Garcés, S. Madurga, and F. Mas. Macromolecular diffusion in crowded media beyond the hard-sphere model. Soft Matter, 14:3105-3114, 2018. doi: 10.1039/C8SM00201K. URL http://dx.doi.org/10.1039/C8SM00201K.

G. E. Briggs and J. B. Haldane. A note on the kinetics of enzyme action. The Biochemical journal, 19(2):338-339, 1925. doi: 10.1042/bj0190338. URL https://pubmed.ncbi.nlm.nih.gov/16743508.

M. Cox and D. Nelson. Lehninger Principles of Biochemistry, volume 5. 01 2000. doi: 10.1007/978-3-662-08289-8.

U. Dieckmann and M. Doebeli. On the origin of species by sympatric speciation. Nature, 400(6742):354-357, 1999. doi: 10.1038/22521. URL https://doi.org/10.1038/22521.

U. Dieckmann and R. Law. The dynamical theory of coevolution: a derivation from stochastic ecological processes. Journal of Mathematical Biology, 34(5):579-612, 1996. doi: 10.1007/BF02409751. URL https://doi.org/10.1007/BF02409751.

K. A. Dill, K. Ghosh, and J. D. Schmit. Physical limits of cells and proteomes. Proceedings of the National Academy of Sciences, 108(44):17876, 11 2011. doi: 10.1073/pnas.1114477108. URL http://www.pnas.org/content/108/44/17876.abstract.

M. Doebeli. A model for the evolutionary dynamics of cross-feeding polymorphisms in microorganisms. Population Ecology, 44(2):59-70, 2002. doi: 10.1007/s101440200008. URL https://doi.org/10.1007/s101440200008.

G. D'Souza, S. Shitut, D. Preussger, G. Yousif, S. Waschina, and C. Kost. Ecology and evolution of metabolic crossfeeding interactions in bacteria. Natural Product Reports, 35(5):455-488, 2018. doi: 10.1039/C8NP00009C. URL http: //dx.doi.org/10.1039/C8NP00009C.

B. Enjalbert, P. Millard, M. Dinclaux, J.-C. Portais, and F. Létisse. Acetate fluxes in escherichia coli are determined by the thermodynamic control of the pta-acka pathway. Scientific Reports, 7(1):42135, 2017. doi: 10.1038/srep42135. URL https://doi.org/10.1038/srep42135.

S. Estrela, J. C. C. Vila, N. Lu, D. Bajic, M. Rebolleda-Gomez, C.-Y. Chang, and A. Sanchez. Metabolic rules of microbial community assembly. bioRxiv, page 2020.03.09.984278, 01 2020. doi: 10.1101/2020.03.09.984278. URL http://biorxiv . org/content/early/2020/03/11/2020.03.09.984278. abstract. 
G. F. Gause. Experimental studies on the struggle for existence : I. mixed population of two species of yeast. Journal of Experimental Biology, 9(4):389-402, 9/7/2021 1932. doi: 10.1242/jeb.9.4.389. URL https://doi.org/10.1242/jeb.9.4. 389.

S. A. H. Geritz, E. Kisdi, G. Meszena, and J. A. J. Metz. Evolutionarily singular strategies and the adaptive growth and branching of the evolutionary tree. Evolutionary Ecology, 12(1):35-57, 1998. doi: 10.1023/A:1006554906681. URL https://doi.org/10.1023/A:1006554906681.

J. E. Goldford, N. Lu, D. Bajić, S. Estrela, M. Tikhonov, A. Sanchez-Gorostiaga, D. Segrè, P. Mehta, and A. Sanchez. Emergent simplicity in microbial community assembly. Science, 361(6401):469, 08 2018. doi: 10.1126/science.aat1168. URL http://science.sciencemag.org/content/361/6401/469. abstract.

B. H. Good, M. J. McDonald, J. E. Barrick, R. E. Lenski, and M. M. Desai. The dynamics of molecular evolution over 60,000 generations. Nature, 551(7678):45-50, 2017. doi: 10.1038/nature24287. URL https://doi.org/10.1038/nature24287.

A. J. Greenberg, S. R. Stockwell, and A. G. Clark. Evolutionary constraint and adaptation in the metabolic network of drosophila. Molecular Biology and Evolution, 25(12):2537-2546, 3/18/2020 2008. doi: 10.1093/molbev/msn205. URL https://doi.org/10.1093/molbev/msn205.

J. B. S. Haldane. Enzymes. Longmans, Green, London, New York, 1930. URL //catalog.hathitrust.org/Record/001580201.

G. Hardin. The competitive exclusion principle. Science, 131 3409:1292-7, 1960.

B. Hasselman. Solve systems of nonlinear equations - package 'nleqslv'. Technical Report Version 3.3.2, 2018.

D. Heckmann, D. C. Zielinski, and B. O. Palsson. Modeling genome-wide enzyme evolution predicts strong epistasis underlying catalytic turnover rates. Nature Communications, 9(1):5270, 2018. doi: 10.1038/s41467-018-07649-1. URL https://doi. org/10.1038/s41467-018-07649-1.

R. Heinrich, S. Schuster, and H. G. Holzhütter. Mathematical analysis of enzymic reaction systems using optimization principles. Eur J Biochem, 201(1):1-21, Oct 1991. ISSN 0014-2956 (Print); 0014-2956 (Linking). doi: 10.1111/j.1432-1033. 1991.tb16251.x.

R. B. Helling, C. N. Vargas, and J. Adams. Evolution of $<$ em $>$ escherichia coli $</$ em $>$ during growth in a constant environment. Genetics, 116(3):349, 07 1987. URL http://www.genetics.org/content/116/3/349.abstract.

R. Hermsen, H. Okano, C. You, N. Werner, and T. Hwa. A growth-rate composition formula for the growth of e. coli on co-utilized carbon substrates. Molecular Systems Biology, 11(4):801, 2021/09/24 2015. doi: https://doi.org/10.15252/msb. 20145537. URL https://doi.org/10.15252/msb.20145537.

J. Hofbauer and K. Sigmund. Adaptive dynamics and evolutionary stability. Applied Mathematics Letters, 3(4):75-79, 1990. doi: https://doi.org/10.1016/0893-9659(90)90051-C. URL https://www.sciencedirect.com/science/article/ pii/089396599090051C.

G. E. Hutchinson. The paradox of the plankton. The American Naturalist, 95(882):137-145, 2021/09/24 1961. doi: 10.1086/ 282171. URL https://doi.org/10.1086/282171.

M. J. Jeffrey, L. R. E., and Z. E. R. The black queen hypothesis: Evolution of dependencies through adaptive gene loss. $m$ Bio, 3(2):e00036-12, 2021/10/13 2012. doi: 10.1128/mBio.00036-12. URL https://doi.org/10.1128/mBio.00036-12.

M. Kafri, E. Metzl-Raz, G. Jona, and N. Barkai. The cost of protein production. Cell Reports, 14(1):22-31, 2016. doi: https:// doi.org/10.1016/j.celrep.2015.12.015. URL https://www.sciencedirect.com/science/article/pii/S221112471501428X. 
H. Kemble, C. Eisenhauer, A. Couce, A. Chapron, M. Magnan, G. Gautier, H. Le Nagard, P. Nghe, and O. Tenaillon. Flux, toxicity, and expression costs generate complex genetic interactions in a metabolic pathway. Science Advances, 6(23): eabb2236, 2021/12/17 . doi: 10.1126/sciadv.abb2236. URL https://doi.org/10.1126/sciadv.abb2236.

A. Kotyk. Mobility of the free and of the loaded monosaccharide carrier in saccharomyces cerevisiae. Biochimica et Biophysica Acta (BBA) - Biomembranes, 135(1):112-119, 1967. doi: https://doi.org/10.1016/0005-2736(67)90013-2. URL http: //www.sciencedirect.com/science/article/pii/0005273667900132.

S. Kryazhimskiy. Emergence and propagation of epistasis in metabolic networks. eLife, 10:e60200, 2021. doi: 10.7554/eLife. 60200. URL https://doi.org/10.7554/eLife.60200.

F. Labourel and E. Rajon. Resource uptake and the evolution of moderately efficient enzymes. Molecular Biology and Evolution, 5/10/2021 2021. doi: 10.1093/molbev/msab132. URL https://doi.org/10.1093/molbev/msab132.

R. E. Lenski, M. R. Rose, S. C. Simpson, and S. C. Tadler. Long-term experimental evolution in escherichia coli. i. adaptation and divergence during 2,000 generations. 138(6):1315-1341, 2021/08/17/ 1991. URL http://www.jstor.org/stable/ 2462549 .

B. R. Levin. Coexistence of two asexual strains on a single resource. Science, 175(4027):1272-1274, 2021/09/08/ 1972. URL http://www. jstor.org/stable/1733726.

X. Li, F. Wu, F. Qi, and D. A. Beard. A database of thermodynamic properties of the reactions of glycolysis, the tricarboxylic acid cycle, and the pentose phosphate pathway. Database, 2011, 9/27/2021 2011. doi: 10.1093/database/bar005. URL https://doi.org/10.1093/database/bar005.

W. Liebermeister, E. Noor, A. Flamholz, D. Davidi, J. Bernhardt, and R. Milo. Visual account of protein investment in cellular functions. Proceedings of the National Academy of Sciences, 111(23):8488, 06 2014. doi: 10.1073/pnas.1314810111. URL http://www.pnas.org/content/111/23/8488. abstract.

M. Lynch and G. K. Marinov. The bioenergetic costs of a gene. Proceedings of the National Academy of Sciences of the United States of America, 112(51):15690-15695, 12 2015. doi: 10.1073/pnas.1514974112. URL http://www.ncbi.nlm.nih.gov/ pmc/articles/PMC4697398/.

J. Meijer, B. van Dijk, and P. Hogeweg. Contingent evolution of alternative metabolic network topologies determines whether cross-feeding evolves. Communications Biology, 3(1):401, 2020. doi: 10.1038/s42003-020-1107-x. URL https://doi.org/ $10.1038 / \mathrm{s} 42003-020-1107-\mathrm{x}$.

R. E. Michod. On the transfer of fitness from the cell to the multicellular organism. Biology and Philosophy, 20(5):967-987, 2005. doi: 10.1007/s10539-005-9018-2. URL https://doi.org/10.1007/s10539-005-9018-2.

P. Millard, B. Enjalbert, S. Uttenweiler-Joseph, J.-C. Portais, F. Létisse, M. Doebeli, and N. Barkai. Control and regulation of acetate overflow in escherichia coli. eLife, 10:e63661, 2021. doi: 10.7554/eLife.63661. URL https://doi.org/10.7554/ eLife.63661.

R. Milo and O. N. Phillips, R. and. Cell Biology by the Numbers. Taylor \& Francis Inc, 2016.

T. D. Niehaus and K. B. Hillmann. Enzyme promiscuity, metabolite damage, and metabolite damage control systems of the tricarboxylic acid cycle. The FEBS Journal, 287(7):1343-1358, 2021/02/18 2020. doi: https://doi.org/10.1111/febs.15284. URL https://doi.org/10.1111/febs.15284.

C. F. Olson-Manning, C.-R. Lee, M. D. Rausher, and T. Mitchell-Olds. Evolution of flux control in the glucosinolate pathway in arabidopsis thaliana. Molecular Biology and Evolution, 30(1):14-23, 3/18/2020 2012. doi: 10.1093/molbev/mss204. URL https://doi.org/10.1093/molbev/mss204. 
M. Orsi, W. E. Sanderson, and J. W. Essex. Permeability of small molecules through a lipid bilayer: A multiscale simulation study. The Journal of Physical Chemistry B, 113(35):12019-12029, 09 2009. doi: 10.1021/jp903248s. URL https://doi. org/10.1021/jp903248s.

K. Peebo, K. Valgepea, A. Maser, R. Nahku, K. Adamberg, and R. Vilu. Proteome reallocation in escherichia coli with increasing specific growth rate. Mol Biosyst, 11(4):1184-1193, Apr 2015. ISSN 1742-2051 (Electronic); 1742-2051 (Linking). doi: $10.1039 / \mathrm{c} 4 \mathrm{mb} 00721 \mathrm{~b}$.

A. Peracchi. The limits of enzyme specificity and the evolution of metabolism. Trends in Biochemical Sciences, 43(12):984996, 2018. doi: https://doi.org/10.1016/j.tibs.2018.09.015. URL https://www.sciencedirect.com/science/article/pii/ S0968000418302007.

T. Pfeiffer, S. Bonhoeffer, and A. E. P. J. Morin. Evolution of cross-feeding in microbial populations. 163(6):E126-E135, 2021/09/06/ 2004. doi: 10.1086/383593. URL http://www.jstor.org/stable/10.1086/383593.

S. Pinhal, D. Ropers, J. Geiselmann, and H. de Jong. Acetate metabolism and the inhibition of bacterial growth by acetate. Journal of bacteriology, 201(13):e00147-19, 06 2019. doi: 10.1128/JB.00147-19. URL https://pubmed.ncbi.nlm.nih.gov/ 30988035.

M. San Roman and A. Wagner. An enormous potential for niche construction through bacterial cross-feeding in a homogeneous environment. PLOS Computational Biology, 14(7):e1006340-, 07 2018. URL https://doi.org/10.1371/journal.pcbi. 1006340 .

M. San Roman and A. Wagner. Acetate and glycerol are not uniquely suited for the evolution of cross-feeding in e. coli. $P L O S$ Computational Biology, 16(11):e1008433-, 11 2020. URL https://doi.org/10.1371/journal.pcbi.1008433.

H. Sheftel, O. Shoval, A. Mayo, and U. Alon. The geometry of the pareto front in biological phenotype space. Ecology and Evolution, 3(6):1471-1483, 2021/08/20 2013. doi: https://doi.org/10.1002/ece3.528. URL https://doi.org/10.1002/ ece3. 528 .

N. W. Smith, P. R. Shorten, E. Altermann, N. C. Roy, and W. C. McNabb. The classification and evolution of bacterial cross-feeding. Frontiers in Ecology and Evolution, 7:153, 2019. ISSN 2296-701X. doi: 10.3389/fevo.2019.00153. URL https://www.frontiersin.org/article/10.3389/fevo.2019.00153.

A. Solopova, J. van Gestel, F. J. Weissing, H. Bachmann, B. Teusink, J. Kok, and O. P. Kuipers. Bet-hedging during bacterial diauxic shift. Proceedings of the National Academy of Sciences, 111(20):7427, 05 2014. doi: 10.1073/pnas.1320063111. URL http://www . pnas.org/content/111/20/7427. abstract.

S. C. Stearns. Trade-offs in life-history evolution. 3(3):259-268, 2021/08/08/ 1989. doi: 10.2307/2389364. URL http: //www.jstor.org.inee.bib.cnrs.fr/stable/2389364.

M. Szenk, K. A. Dill, and A. M. R. de Graff. Why do fast-growing bacteria enter overflow metabolism? testing the membrane real estate hypothesis. Cell Systems, 5(2):95-104, 2017. doi: https://doi.org/10.1016/j.cels.2017.06.005. URL https: //www.sciencedirect.com/science/article/pii/S2405471217302338.

D. S. Treves, S. Manning, and J. Adams. Repeated evolution of an acetate-crossfeeding polymorphism in long-term populations of escherichia coli. Molecular Biology and Evolution, 15(7):789-797, 9/24/2021 1998. doi: 10.1093/oxfordjournals.molbev. a025984. URL https://doi.org/10.1093/oxfordjournals.molbev. a025984.

G. N. Vemuri, E. Altman, D. P. Sangurdekar, A. B. Khodursky, and M. A. Eiteman. Overflow metabolism in escherichia coli during steady-state growth: transcriptional regulation and effect of the redox ratio. Appl Environ Microbiol, 72(5):36533661, May 2006. ISSN 0099-2240 (Print); 1098-5336 (Electronic); 0099-2240 (Linking). doi: 10.1128/AEM.72.5.3653-3661. 2006. 
A. Wagner. Energy Constraints on the Evolution of Gene Expression. Molecular Biology and Evolution, 22(6):1365-1374, 03 2005. ISSN 0737-4038. doi: 10.1093/molbev/msi126. URL https://doi.org/10.1093/molbev/msi126.

X. Wang, K. Xia, X. Yang, and C. Tang. Growth strategy of microbes on mixed carbon sources. Nature Communications, 10 (1):1279, 2019. doi: 10.1038/s41467-019-09261-3. URL https://doi.org/10.1038/s41467-019-09261-3.

K. M. Wright and M. D. Rausher. The evolution of control and distribution of adaptive mutations in a metabolic pathway. Genetics, 184(2):483, 02 2010. doi: 10.1534/genetics.109.110411. URL http://www.genetics.org/content/184/2/483. abstract.

S. Wright. The genetical structure of populations. Annals of Eugenics, 15(1):323-354, 2021/09/25 1949. doi: https://doi.org/ 10.1111/j.1469-1809.1949.tb02451.x. URL https://doi.org/10.1111/j.1469-1809.1949.tb02451.x.

K. Zhuang, G. N. Vemuri, and R. Mahadevan. Economics of membrane occupancy and respiro-fermentation. Molecular Systems Biology, 7(1):500, 2021/08/19 2011. doi: https://doi.org/10.1038/msb.2011.34. URL https://doi.org/10.1038/ $\mathrm{msb} .2011 .34$. 